\title{
A New Hybrid Generalized Selection Combining Scheme and Its Performance over Fading Channels
}

\author{
Yunxia Chen and Chintha Tellambura \\ Department of Electrical and Computer Engineering \\ University of Alberta, Edmonton, AB, Canada T6G 2V4 \\ Ph:1 7804927228 Fax:1 7804921811 \\ Email: \{yunxia,chintha\}@ece.ualberta.ca
}

\begin{abstract}
Emerging very high-data-rate wireless communications requires that traditional diversity systems be adapted so that some performance is sacrificed for complexity reduction. With this goal in mind, we investigate the recently-developed absolute threshold generalized selection combining (AT-GSC). We show that AT-GSC has poor bit-error rate (BER) performance when the average branch signal-to-noise ratio (SNR) is comparably lower than the preset threshold. This paper therefore develops a new diversity combining scheme, referred to as switching GSC (SGSC). This scheme combines all branches whose SNR's exceed a preset threshold and if all the branches drop below the threshold, the output is the single branch with the maximum SNR. We prove that for any two-dimensional amplitude/phase linear modulation schemes, the symbol error rate (SER) of S-GSC lies between those of maximal ratio combining (MRC) and selection combing (SC). Importantly, the complexity of S-GSC is only slightly above that of AT-GSC. We derive the moment generating function (mgf) of the S-GSC output SNR over independent fading channels. For identically and independently distributed (i.i.d.) Rayleigh fading channels, we also derive the output probability density function (pdf) and cumulative distribution function (cdf) and analyze the error rate and the outage probability performance of S-GSC. Higher order moments of the S-GSC output SNR are also derived. These theoretical results are sufficient to completely characterize the performance of S-GSC and enable one to compare S-GSC with conventional diversity schemes.
\end{abstract}

\section{INTRODUCTION}

Diversity techniques have long been employed to combat the effect of multi-path fading on wireless communication systems. Recent interest on emerging high-data-rate wireless systems has led to a renewal of the subject. Classical diversity techniques include MRC, equal gain combining (EGC) and SC. An $L$-branch MRC performs the best with the highest complexity and SC, on the other hand, performs the worst comparatively with the least complexity. This motivates the development of other diversity schemes whose complexity and performance lie between these two extreme cases. Therefore, several suboptimum hybrid diversity combining schemes that achieve a tradeoff between performance and implementation complexity have recently been proposed and studied in the literature. Generalized selection combining (GSC) [1]-[5], initially proposed by Kong et al. [5], combines $M(1 \leq M \leq$ $L)$ branches with the largest instantaneous SNR's out of $L$ branches. We shall use notation $\operatorname{GSC}(L, M)$ and remark that the special cases $\operatorname{GSC}(L, L)$ and $\operatorname{GSC}(L, 1)$ are MRC and
$\mathrm{SC}$, respectively. Since $M$ is fixed, $\operatorname{GSC}(L, M)$ has a fixed processing complexity. Moreover, choosing an appropriate $M$ for various fading channels may be difficult [2].

Two further developments in GSC have been proposed and analyzed [2], [6], [7]. The key idea is to allow $M$ vary by discarding highly faded branch signals dynamically depending on the received branch signal strength profile. Sulyman and Kousa [6] and Yue [2] present a normalized threshold GSC (NT-GSC) scheme, which combines the branches whose relative branch strength (the ratio of the SNR of each branch to the maximum SNR) exceeds a fixed normalized threshold $T(0<T \leq 1)$. Yue [2] presents AT-GSC, which combines all the branches with SNR's above a fixed threshold (say, $\left.\gamma_{t h}\right)$. Simon and Alouini [7] provide a comprehensive performance analysis of both ATGSC and NT-GSC over independent fading channels. These two schemes are more flexible than $\operatorname{GSC}(L, M)$ in situations where the receiver is presented with a large number of multipath components or diversity branches, such as RAKE receivers for ultra-wideband (UWB) spread-spectrum signals. Note that they both are suboptimal schemes and their performance cannot be better than that of MRC.

This paper is therefore motivated by an apparent anomaly observed in [7], where AT-GSC is found to have better BER performance than MRC. This has prompted us to investigate the AT-GSC performance as a function of the preset threshold $\left(\gamma_{t h}\right)$. The counter-intuitive result in [7] is due to the way the BER is computed. The error state of AT-GSC in which no branches contribute to the combiner output is ignored in the analysis of [7]. This error state occurs when all the branch SNR's fall below $\gamma_{t h}$ simultaneously. When it is taken into consideration, the ATGSC performance degrades monotonically with the increasing $\gamma_{t h}$. In fact, AT-GSC performs worse than SC when the average branch SNR is comparably lower than $\gamma_{t h}$. This raises the question if there is a way to improve the AT-GSC performance.

We therefore propose a new scheme called S-GSC, in which all the branches whose SNR's exceed $\gamma_{t h}$ are combined to form the output, and if all the branch SNR's fall below $\gamma_{t h}$, the maximum branch SNR is applied to the combiner. Thus, SGSC eliminates the error state that presents in AT-GSC. We show that S-GSC outperforms both conventional SC and ATGSC. It reduces to classical MRC and SC when $\gamma_{t h}=0$ and $\gamma_{t h}=\infty$, respectively. Moreover, S-GSC is only slightly 
more complex than AT-GSC in terms of the average number of diversity branches combined.

The paper is organized as follows. Section 2 analyzes the effect of the threshold on the AT-GSC performance. Section 3 develops a new diversity combining scheme and also derives its output mgf, pdf and cdf over independent fading channels. In Section 4, the average error rates of various modulation schemes, outage probability and other output quality measures are derived. Section 5 presents numerical results to compare the new scheme with other combining techniques and concludes the paper.

\section{AT-GSC PERformanCE AS A FUnCTION OF THRESHOLD}

Consider a diversity combiner with $L$ branches. Let $\gamma_{l}(l=$ $1,2, \cdots, L)$ denotes the instantaneous SNR of the $l$-th branch. In AT-GSC, all the branches whose instantaneous SNR's exceed a fixed threshold are combined to form the output [2], [7], which may be written as

$$
\gamma_{a t}= \begin{cases}0, & \text { if all } \gamma_{l}<\gamma_{t h}, \\ \sum_{\gamma_{l} \geq \gamma_{t h}} \gamma_{l}, & \text { otherwise }\end{cases}
$$

where $\gamma_{t h}$ is a preset threshold.

In [7], the error state in which no branches contribute to the combiner output was subtracted from the expression for the BER of AT-GSC. However, we expect that as the threshold $\left(\gamma_{t h}\right)$ increases, fewer branches will be combined as the output, which results in higher BER. We prove this intuitive notion rigorously by using the mgf of the AT-GSC output. For i.i.d. Rayleigh fading channels, the mgf of the AT-GSC output is given by [7]

$$
M_{a t}(s)=E\left(e^{-s \gamma}\right)=\left[1-e^{-\frac{\gamma_{t h}}{\bar{\gamma}}}+\frac{e^{-\frac{\gamma_{t h}}{\bar{\gamma}}(1+s \bar{\gamma})}}{1+s \bar{\gamma}}\right]^{L}
$$

where $E(x)$ denotes the average of $x$ and $\bar{\gamma}$ is the average SNR per branch. For any real value $s>0$, we know that $M_{a t}(s)>0$ and

$$
\begin{aligned}
\frac{\partial M_{a t}(s)}{\partial \gamma_{t h}}= & L\left[1-e^{-\frac{\gamma_{t h}}{\bar{\gamma}}}+\frac{e^{-\frac{\gamma_{t h}}{\gamma}(1+s \bar{\gamma})}}{1+s \bar{\gamma}}\right]^{L-1} \\
& \times\left[\frac{1}{\bar{\gamma}} e^{-\frac{\gamma_{t h}}{\bar{\gamma}}}\left(1-e^{-s \gamma_{t h}}\right)\right]>0 .
\end{aligned}
$$

Hence $M_{a t}(s)$ monotonically increases with $\gamma_{t h}$. Applying the mgf technique [8], [9], we may compute the SER of any twodimensional amplitude/phase linear modulation using

$$
\bar{P}_{s}=\frac{1}{2 \pi} \sum_{k=1}^{S} W_{k} \int_{0}^{\eta_{k}} M_{a t}\left(\frac{a_{k} \sin ^{2}\left(\varphi_{k}\right)}{\sin ^{2}\left(\theta+\varphi_{k}\right)}\right) d \theta
$$

where $S$ is the total number of signal points or decision subregions, $W_{k}>0, a_{k}>0, \eta_{k}$ and $\varphi_{k}$ are parameters relating to decision subregion $k$ and they are independent of the instantaneous SNR $\gamma$. Combining (4) with (3), we readily find that the average SER increases with $\gamma_{t h}$.
Fig. 1 shows that for the fixed average branch SNR $(\bar{\gamma})$ and the number of diversity branches $(L)$, the BER $(\bar{P})$ of binary phase-shift-keying (BPSK) with AT-GSC increases dramatically as the preset threshold $\left(\gamma_{t h}\right)$ increases. It approaches $1 / 2$ in the worst case. This makes AT-GSC unsuitable for practical applications and motivates improvements for AT-GSC.

\section{New Hybrid GSC COMbining SCHEmes}

To mitigate the worsening system performance as the threshold $\left(\gamma_{t h}\right)$ increases, we present a new hybrid GSC schemes, S-GSC.

\section{A. S-GSC over Independent Fading Channels}

In the new hybrid GSC scheme, S-GSC, all the branches with SNR's exceeding a fixed preset threshold $\left(\gamma_{t h}\right)$ are combined as the output. If no branch SNR exceeds $\gamma_{t h}$, the branch with the largest SNR is selected. S-GSC switches therefore between AT-GSC and SC depending on how many branch SNR's exceed $\gamma_{t h}$. Consequently, the combiner output can be written as

$$
\gamma_{s g}= \begin{cases}\max \left(\gamma_{l}\right), & \text { if all } \gamma_{l}<\gamma_{t h}, \\ \sum_{\gamma_{l} \geq \gamma_{t h}} \gamma_{l}, & \text { otherwise. }\end{cases}
$$

For performance analysis, the pdf and/or the mgf of the SGSC output $\left(\gamma_{s g}\right)$ is required. The pdf of $\gamma_{s g}$ can be expressed in terms of the output pdf of SC $p_{s c}(\gamma)$ and the output pdf of AT-GSC $p_{a t}(\gamma)$ as

$$
\begin{aligned}
p_{s g}(\gamma)= & \frac{p_{s c}\left(\gamma \mid 0 \leq \gamma<\gamma_{t h}\right)}{\int_{0}^{\gamma_{t h}} p_{s c}(\gamma) d \gamma} \operatorname{Pr}\left(\text { all } \gamma_{l}<\gamma_{t h}\right) \\
& +\frac{p_{a t}\left(\gamma \mid \gamma \geq \gamma_{t h}\right)}{\int_{\gamma_{t h}}^{\infty} p_{a t}(\gamma) d \gamma} \operatorname{Pr}\left(\text { at least one } \gamma_{l} \geq \gamma_{t h}\right) \\
= & p_{s c}(\gamma)\left[u(\gamma)-u\left(\gamma-\gamma_{t h}\right)\right]+p_{a t}(\gamma) u\left(\gamma-\gamma_{t h}\right)
\end{aligned}
$$

where $u(x)$ is the unit step function

$$
u(x)= \begin{cases}0, & x<0 \\ 1, & x \geq 0\end{cases}
$$

Using the previous analysis of SC [10] and AT-GSC [7], we can readily derive a general expression for the mgf of the S-GSC output over independent fading channels:

$$
\begin{aligned}
& M_{s g}(s)=\sum_{l=1}^{L}\left[\int_{0}^{\gamma_{t h}}\left(e^{-s \gamma} p_{l}(\gamma) \prod_{i=1, i \neq l}^{L} F_{i}(\gamma)\right) d \gamma\right] \\
& +\prod_{l=1}^{L}\left[F_{l}\left(\gamma_{t h}\right)+\int_{\gamma_{t h}}^{\infty} p_{l}(\gamma) e^{-s \gamma} d \gamma\right]-\prod_{l=1}^{L} F_{l}\left(\gamma_{t h}\right)
\end{aligned}
$$

where $p_{l}(\gamma)$ and $F_{l}(\gamma)$ are the pdf and the cdf of the $l$-th branch SNR. For example, the mgf of the S-GSC output SNR over independent (not necessarily identical) Rayleigh fading 
channels can be obtained as

$$
\begin{aligned}
M_{s g}(s) & =\sum_{b_{1}+\cdots+b_{L}=1}^{L}(-1)^{b_{1}+\cdots+b_{L}+1}\left(\sum_{l=1}^{L} \frac{b_{l}}{\bar{\gamma}_{l}}\right) \\
& \times \frac{1-e^{-\left(s+\sum_{l=1}^{L} \frac{b_{l}}{\bar{\gamma}_{l}}\right) \gamma_{t h}}}{s+\sum_{l=1}^{L} \frac{b_{l}}{\bar{\gamma}_{l}}}\left[1-e^{-\frac{\gamma_{t h}}{\bar{\gamma}_{l}}}\right] \\
& +\prod_{l=1}^{L}\left\{1-e^{-\frac{\gamma_{t h}}{\bar{\gamma}_{l}}}+\frac{e^{-\left(s+\frac{1}{\gamma_{l}}\right) \gamma_{t h}}}{1+s \bar{\gamma}_{l}}\right\} .
\end{aligned}
$$

Recall that two limiting cases of S-GSC.

1. When $\gamma_{t h}=0$, the output mgf (8) can be simplified to

$$
M_{s g}(s)=\prod_{l=1}^{L}\left[\int_{0}^{\infty} p_{l}\left(\gamma_{l}\right) e^{-s \gamma_{l}} d \gamma_{l}\right]
$$

which is equivalent to the case of MRC.

2. When $\gamma_{t h}=\infty$, the output mgf (8) reduces to

$$
M_{s g}(s)=\sum_{l=1}^{L}\left[\int_{0}^{\infty}\left(e^{-s \gamma_{s}} p_{l}\left(\gamma_{s}\right) \prod_{i=1, i \neq l}^{L} F_{i}\left(\gamma_{s}\right)\right) d \gamma_{s}\right]
$$

which is equivalent to the case of SC. These special cases reaffirm the correctness of (8).

\section{B. S-GSC over i.i.d. Rayleigh Fading Channels}

For the special case of i.i.d. Rayleigh fading channels, the mgf (8) of the S-GSC output reduces to

$$
\begin{aligned}
M_{s g}(s) & =\sum_{n=1}^{L}\left(\begin{array}{l}
L \\
n
\end{array}\right)(-1)^{n+1} n \frac{1-e^{-\frac{\gamma_{t h}}{\bar{\gamma}}(n+\bar{\gamma} s)}}{n+\bar{\gamma} s} \\
& +\sum_{l=1}^{L}\left(\begin{array}{l}
L \\
l
\end{array}\right)\left[1-e^{-\frac{\gamma_{t h}}{\bar{\gamma}}}\right]^{L-l}\left[\frac{e^{-\frac{\gamma_{t h}}{\bar{\gamma}}(1+\bar{\gamma} s)}}{1+\bar{\gamma} s}\right]^{l} .
\end{aligned}
$$

Note that the first part of (12) accounts for the event that all branch SNR's drop below the threshold and S-GSC switches to $\mathrm{SC}$, and the second part corresponds to the case where $l$ branches $(l=1, \ldots, L)$ exceed the threshold.

Appendix shows that for a certain $\bar{\gamma}$ and real $s>0$, the output mgf increases monotonically with $\gamma_{t h}$. Hence, we can prove that the error rate performance of S-GSC is upper bounded by MRC and lower bounded by SC.

The output pdf is the inverse Laplace transform of the output mgf (12), which can be derived as

$$
\begin{aligned}
p_{s g}(x) & =\frac{L}{\bar{\gamma}}\left(1-e^{-\frac{x}{\bar{\gamma}}}\right)^{L-1} e^{-\frac{x}{\bar{\gamma}}}\left[1-u\left(x-\gamma_{t h}\right)\right] \\
& +\frac{1}{\bar{\gamma}} \sum_{l=1}^{L}\left(\begin{array}{c}
L \\
l
\end{array}\right) \frac{1}{(l-1) !}\left(1-e^{-\frac{\gamma_{t h}}{\bar{\gamma}}}\right)^{L-l} e^{-\frac{x}{\bar{\gamma}}} \\
& \times\left(\frac{x-l \gamma_{t h}}{\bar{\gamma}}\right)^{l-1} u\left(x-l \gamma_{t h}\right) .
\end{aligned}
$$

Integrating (13) over $x$ yields the output cdf to be

$$
\begin{aligned}
F_{s g}(x) & =\left(1-e^{-\frac{x}{\bar{\gamma}}}\right)^{L}\left[1-u\left(x-\gamma_{t h}\right)\right]+\left(1-e^{-\frac{\gamma_{t h}}{\bar{\gamma}}}\right)^{L} \\
& \times u\left(x-\gamma_{t h}\right) \sum_{m=1}^{\min \left\{L,\left\lfloor x / \gamma_{t h}\right\rfloor\right\}}\left(\begin{array}{c}
L \\
m
\end{array}\right)\left(1-e^{-\frac{\gamma_{t h}}{\bar{\gamma}}}\right)^{L-m} \\
& \times\left[1-e^{-\frac{x-m \gamma_{t h}}{\bar{\gamma}}} \sum_{j=0}^{m-1} \frac{\left(\frac{x-m \gamma_{t h}}{\bar{\gamma}}\right)^{m-1-j}}{(m-1-j) !}\right] .
\end{aligned}
$$

\section{PERformance AnAlysis}

In this section, the average error rates, outage probability and several output quality measures of S-GSC over i.i.d. Rayleigh fading channels are presented. We assume that channel fading is flat and changing slowly enough so that the channel parameters remain constant for the duration of signaling interval. Our results are based on the mgf expression (12). For other independent fading channels, the S-GSC performance can be readily evaluated using (8). For brevity, we do not develop such results here.

\section{A. Average error rate}

The BER or the SER is one of the most commonly used performance criterion of digital communication systems. The mgf approach [8], [9] can be readily employed for evaluating the average error rates of various kinds of modulation schemes using S-GSC.

1) BPSK and binary frequency-shift-keying (BFSK): The conditional error probability (CEP) for coherent BPSK and BFSK can be expressed as

$$
P_{b}(\gamma)=Q(\sqrt{2 \alpha \gamma})=\frac{1}{\pi} \int_{0}^{\pi / 2} \exp \left(-\frac{\alpha \gamma}{\sin ^{2} \theta}\right) d \theta
$$

where $\alpha=1$ for BPSK and $\alpha=\frac{1}{2}$ for BFSK. The advantage of these exponential representations for analysis over fading channels is that the final average error rates can be expressed strictly in terms of the mgf. Therefore, the BER can be readily shown as [3]

$$
\bar{P}_{b}=\frac{1}{\pi} \int_{0}^{\pi / 2} M_{s g}\left(\frac{\alpha}{\sin ^{2} \theta}\right) d \theta .
$$

Using the mgf of the S-GSC output (12), we obtain the BER of binary coherent signaling with S-GSC as

$$
\begin{aligned}
\bar{P}_{b} & =Q\left(\sqrt{2 \alpha \gamma_{t h}}\right)\left[\left(1-e^{-\frac{\gamma_{t h}}{\bar{\gamma}}}\right)^{L}-1\right]+\sum_{n=1}^{L}\left(\begin{array}{c}
L \\
n
\end{array}\right)(-1)^{n} \\
& \times \sqrt{\frac{\alpha \bar{\gamma}}{\alpha \bar{\gamma}+n}}\left[\frac{1}{2}-Q\left(\sqrt{\frac{2 \gamma_{t h}(n+\alpha \bar{\gamma})}{\bar{\gamma}}}\right)\right]+\frac{1}{2}+\frac{1}{\pi} \\
& \times \sum_{l=1}^{L}\left(\begin{array}{l}
L \\
l
\end{array}\right)\left[1-e^{-\frac{\gamma_{t h}}{\bar{\gamma}}}\right]^{L-l} \int_{0}^{\pi / 2}\left\{\frac{e^{-\frac{\gamma_{t h}}{\bar{\gamma}}\left(1+\frac{\alpha \bar{\gamma}}{\sin ^{2} \theta}\right)}}{1+\frac{\alpha \bar{\gamma}}{\sin ^{2} \theta}}\right\}^{l} d \theta
\end{aligned}
$$

where $Q(x)$ is the area under the tail of the Gaussian pdf and defined as [11, Eq. (2-1-97)]. Since (17) is a simple, finite-range 
integral of exponential and trigonometric functions, it can easily be evaluated numerically using any common mathematical software such as Matlab.

2) Non-coherent binary frequency-shift-keying (NCFSK) and binary differential phase-shift-keying (DPSK): The CEP for NCFSK and DPSK is given by [11]

$$
P_{b}(\gamma)=\frac{1}{2} e^{-\alpha \gamma}
$$

where $\alpha=1$ for BPSK and $\alpha=\frac{1}{2}$ for BFSK. Hence, the corresponding BER is

$$
\begin{aligned}
\bar{P}_{b} & =\frac{1}{2} M_{s g}(\alpha)=\sum_{n=1}^{L}\left(\begin{array}{c}
L \\
n
\end{array}\right)(-1)^{n+1} \frac{1-e^{-\frac{\gamma_{t h}}{\bar{\gamma}}(n+\alpha \bar{\gamma})}}{2(n+\alpha \bar{\gamma})} \\
& +\frac{1}{2} \sum_{l=1}^{L}\left(\begin{array}{c}
L \\
l
\end{array}\right)\left[1-e^{-\frac{\gamma_{t h}}{\bar{\gamma}}}\right]^{L-l}\left[\frac{e^{-\frac{\gamma_{t h}}{\gamma}(1+\alpha \bar{\gamma})}}{1+\alpha \bar{\gamma}}\right]^{l} .
\end{aligned}
$$

Following the procedure above, we can write down formulas of average for other modulation schemes with S-GSC. For brevity, we omit these results and more details can be found in [12].

\section{B. Outage Probability}

The outage probability is another commonly used performance measure of digital communication systems. The outage is defined as the probability that the output instantaneous SNR $\gamma$ falls below a certain given threshold $\gamma_{T}$. The outage relates to the cdf as follows:

$$
P_{\text {out }}=\operatorname{Pr}\left(0 \leq \gamma \leq \gamma_{T}\right)=F_{s g}\left(\gamma_{T}\right) .
$$

By evaluating the output cdf (14) at $\gamma_{T}$, we immediately obtain the outage probability for S-GSC as

$$
\begin{aligned}
P_{\text {out }} & =\left(1-e^{-\frac{\gamma_{T}}{\bar{\gamma}}}\right)^{L}\left[1-u\left(\gamma_{T}-\gamma_{t h}\right)\right]+\left(1-e^{-\frac{\gamma_{t h}}{\bar{\gamma}}}\right)^{L} \\
& \times u\left(\gamma_{T}-\gamma_{t h}\right) \sum_{m=1}^{\min \left\{L,\left\lfloor\gamma_{T} / \gamma_{t h}\right\rfloor\right\}}\left(\begin{array}{c}
L \\
m
\end{array}\right)\left(1-e^{-\frac{\gamma_{t h}}{\bar{\gamma}}}\right)^{L-m} \\
& \times\left[1-e^{-\frac{\gamma_{T}-m \gamma_{t h}}{\bar{\gamma}}} \sum_{j=0}^{m-1} \frac{\left(\frac{\gamma_{T}-m \gamma_{t h}}{\bar{\gamma}}\right)^{m-1-j}}{(m-1-j) !}\right] .
\end{aligned}
$$

\section{Other Output Quality Indicators}

The mean output SNR of a diversity combiner is sometimes used as a comparative performance measure. Using the $\mathrm{mgf}$ of the S-GSC output (8), the moments of the output SNR can readily be determined. The mean output SNR is therefore obtained as

$$
\begin{aligned}
\bar{\gamma}_{s g} & =E\left(\gamma_{s g}\right)=-\left.\frac{d M_{s g}(s)}{d s}\right|_{s=0} \\
& =\bar{\gamma} \sum_{n=1}^{L}\left(\begin{array}{l}
L \\
n
\end{array}\right)\left\{(-1)^{n-1}\left(\frac{1}{n}\right)+e^{-\frac{n \gamma_{t h}}{\gamma}}\left[(-1)^{n}\right.\right. \\
& \left.\left.\left(\frac{1}{n}+\frac{\gamma_{t h}}{\bar{\gamma}}\right)+n\left(1-e^{-\frac{\gamma_{t h}}{\bar{\gamma}}}\right)^{L-l}\left(1+\frac{\gamma_{t h}}{\bar{\gamma}}\right)\right]\right\} .
\end{aligned}
$$

We also obtain the second moment of the output SNR as

$$
\begin{aligned}
\overline{\gamma_{s g}^{2}} & =\left.\frac{d^{2} M_{s g}(s)}{d s^{2}}\right|_{s=0}=\bar{\gamma}^{2} \sum_{n=1}^{L}\left(\begin{array}{c}
L \\
n
\end{array}\right)\left\{(-1)^{n-1} \frac{2}{n^{2}}\right. \\
& +e^{-\frac{n \gamma_{t h}}{\gamma}}\left[(-1)^{n}\left(\frac{2}{n^{2}}+\frac{2 \gamma_{t h}}{n \bar{\gamma}}+\left(\frac{\gamma_{t h}}{\bar{\gamma}}\right)^{2}\right)\right. \\
& \left.\left.+n\left(1-e^{-\frac{\gamma_{t h}}{\bar{\gamma}}}\right)^{L-l}\left(1+l\left(1+\frac{\gamma_{t h}}{\bar{\gamma}}\right)^{2}\right)\right]\right\} .
\end{aligned}
$$

More generally, we obtain higher order moments as $\nu_{n}=$ $E\left(\gamma_{s g}^{n}\right)=\left.(-1)^{n} \frac{d^{n} M_{s g}(s)}{d s^{n}}\right|_{s=0}$. Central moments can then be obtained as $\mu_{k}=E\left[\left(\gamma_{s g}-\bar{\gamma}_{s g}\right)^{k}\right]=\sum_{i=0}^{k}\left(\begin{array}{c}k \\ i\end{array}\right) \nu_{i}\left(-\bar{\gamma}_{s g}\right)^{k-i}$.

The amount of fading (AF) is often used to describe the severity of the channel fading [13]. Alouini and Simon [14] derive closed-form expressions for the AF's of dual diversity combiners over correlated log-normal fading channels. Win and Winters [15] use the square value of the AF (a.k.a. Karl Pearson's coefficient of variation) to assess the effectiveness of GSC scheme over Rayleigh fading channels. Using (22) and (23), the AF of S-GSC over i.i.d. Rayleigh fading channels becomes

$$
\mathrm{AF}=\frac{\mu_{2}}{\nu_{1}}=\frac{\overline{\gamma_{s g}^{2}}}{\bar{\gamma}_{s g}}-1
$$

\section{Complexity Comparison}

The average number of combined branches $\bar{L}_{c}$ may be considered as a measure of the implementation complexity of a diversity combiner.

In S-GSC, the probability that $L_{c}=l$ out of $L$ branches are combined as the output is

$\operatorname{Pr}\left\{L_{c}=l\right\}=\left\{\begin{array}{c}{\left[F_{s g}\left(\gamma_{t h}\right)\right]^{L}+L\left[1-F_{s g}\left(\gamma_{t h}\right)\right]\left[F_{s g}\left(\gamma_{t h}\right)\right]^{L-1}} \\ \text { for } l=1, \\ \left(\begin{array}{l}L \\ l\end{array}\right)\left[F_{s g}\left(\gamma_{t h}\right)\right]^{L-l}\left[1-F_{s g}\left(\gamma_{t h}\right)\right]^{l} \\ \text { for } 2 \leq l \leq L .\end{array}\right.$

Thus, the average number of branches combined is

$$
\bar{L}_{c}=\sum_{l=1}^{L} l \operatorname{Pr}\left\{L_{c}=l\right\}=1+(L-1) e^{-\frac{\gamma_{t h}}{\gamma}} .
$$

Let us compare the complexity of different diversity combiners by listing the average number of combined branches for each:

$$
\bar{L}_{c}= \begin{cases}L, & \text { MRC, } \\ 1, & \text { SC, } \\ 1+(L-1) e^{-\frac{\gamma_{t h}}{\gamma}}, & \text { S-GSC, } \\ L e^{-\frac{\gamma_{t h}}{\gamma}}, & \text { AT-GSC } .\end{cases}
$$

Eq. (27) shows that MRC is the most complicated scheme, while SC is the simplest one. As the threshold $\gamma_{t h}$ increases, the average number of combined branches $\bar{L}_{c}$ of S-GSC approaches to 1 and S-GSC reduces to SC. As $\gamma_{t h}$ decreases, $\bar{L}_{c}$ of SGSC approaches to $L$ and S-GSC approaches MRC. S-GSC 
combines only $1-e^{-\frac{\gamma_{t h}}{\bar{\gamma}}}<1$ more branches than AT-GSC. The complexity of S-GSC is comparable to that AT-GSC when the diversity order is large.

\section{NumericAl RESUlts}

Several numerical results are presented to illustrate the performance of balanced S-GSC over i.i.d. Rayleigh fading channels. In all the figures $\gamma_{t h}^{*}=\gamma_{t h} / \bar{\gamma}$ is the threshold $\left(\gamma_{t h}\right)$ normalized by the average branch SNR $(\bar{\gamma})$.

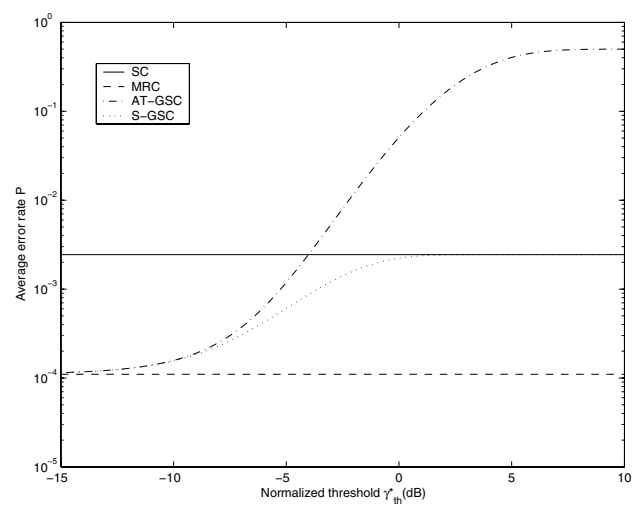

Fig. 1. Comparison of the effect of threshold on the BER of BPSK with S-GSC, AT-GSC, MRC and SC; $\bar{\gamma}=5 \mathrm{~dB}, L=5$.

Fig. 1 shows the BER of BPSK with several diversity schemes as a function of the normalized threshold $\gamma_{t h}^{*}$. Clearly, the MRC and SC performance does not change with $\gamma_{t h}^{*}$. However, the AT-GSC and S-GSC performance is highly dependent on the threshold. As the threshold increases, AT-GSC performs very poorly. When $\gamma_{t h}^{*} \gg 1$ (the threshold $\gamma_{t h}$ is much higher than the average SNR $\bar{\gamma}$ ), the BER of AT-GSC approaches $1 / 2$. Clearly, as the threshold increases, the probability that no branch is selected increases. The performance of S-GSC is less sensitive to the threshold compared to that of AT-GSC. When the threshold is low, S-GSC performs as well as ATGSC. However, for high threshold values, the BER of S-GSC approaches that of SC, which is much lower than that of ATGSC.

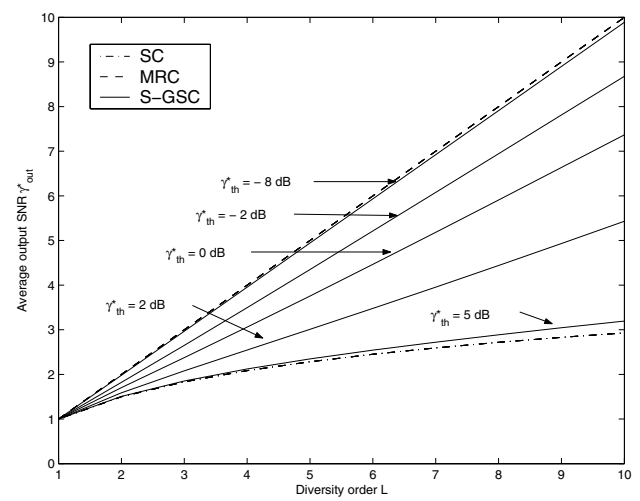

Fig. 2. Comparison of mean output SNR of S-GSC with MRC and SC, $L$ is diversity order, normalized mean output SNR $\gamma_{\text {out }}^{*}=\bar{\gamma}_{\text {out }} / \bar{\gamma}$.
Fig. 2 compares the normalized mean output SNR $\gamma_{\text {out }}^{*}=$ $\bar{\gamma}_{\text {out }} / \bar{\gamma}$ achieved by different diversity schemes. As expected, the mean output SNR of MRC increases linearly with the increasing diversity order $L$ while for SC, it increases much more slowly. This observation agrees with the result in [16]. The mean output SNR of S-GSC is upper and lower bounded by that of MRC and SC, respectively. As the threshold $\gamma_{t h}$ decreases (S-GSC behaves more like MRC), the mean output SNR of S-GSC increases and so does the rate at which it increases. Therefore, lowering the threshold improves the mean output SNR, especially for a higher diversity order. For example, when the threshold changes from $5 \mathrm{~dB}$ to $-8 \mathrm{~dB}$, the mean output SNR improves nearly $2 \mathrm{~dB}$ at $L=4$ and $7 \mathrm{~dB}$ at $L=10$.

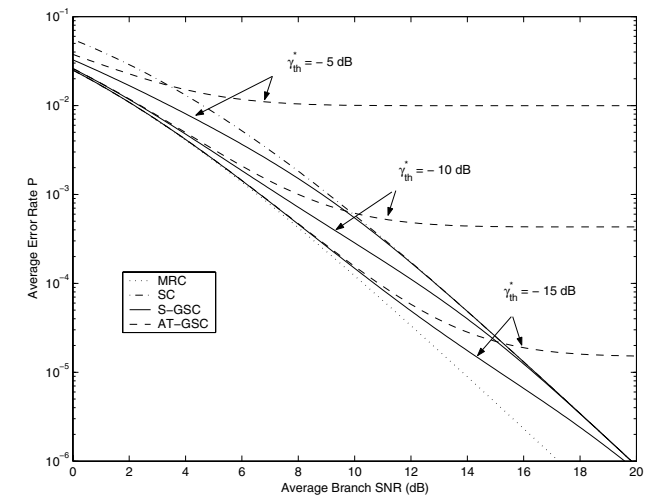

Fig. 3. Comparison of BER of BPSK with S-GSC, AT-GSC, MRC and SC; $L=3$.

The BER of BPSK with different diversity combiners is shown in Fig. 3. As expected, MRC performs the best and AT-GSC performs even worse than SC when the threshold $\gamma_{t h}$ is large. This is because high threshold sends AT-GSC to the error state (the event that all the branch SNR's drop below the threshold), which results in significantly high error rate. However, S-GSC outperforms both SC and AT-GSC.

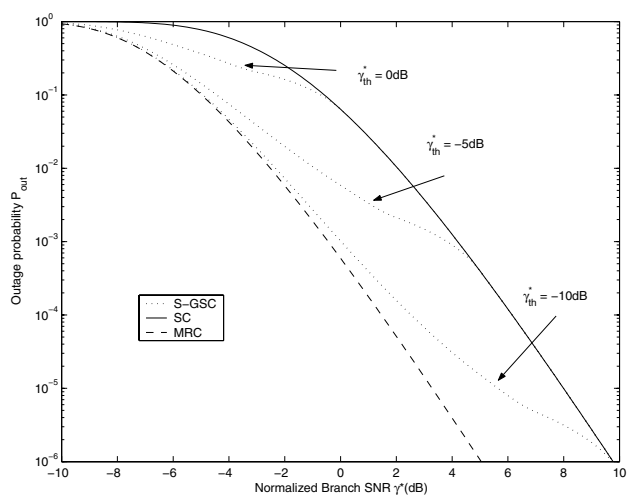

Fig. 4. Comparison of outage probability of S-GSC, SC and MRC; $L=6$, normalized branch SNR $\gamma^{*}=\bar{\gamma} / \gamma_{T}$.

Fig. 4 compares the outage probability of S-GSC with those of SC and MRC. The outage probability of S-GSC approaches 
that of MRC when the threshold is lower than the average SNR. As the threshold increases, S-GSC performs close to SC.

In conclusion, we have shown that AT-GSC performs poorly as the preset threshold increases. To improve system performance, we have presented a new combining scheme, called SGSC, which is a hybrid of SC and AT-GSC. We also developed theoretical performance results for S-GSC, which are general enough to handle any independent fading models. Results show that S-GSC outperforms both SC and AT-GSC with the same threshold. The performance of S-GSC lies between that of MRC and SC. The number of branches contributing to the output of S-GSC is not fixed, but varies corresponding to the channel fading conditions. Hence, S-GSC is flexible enough to provide a tradeoff between system complexity and performance. Finally, an interesting research problem would be to analyze the S-GSC performance over correlated fading channels.

\section{ACKNOWLEDGMENT}

This work has been supported in part by NSERC and iCORE.

\section{APPENDIX}

In this appendix, we prove that the output mgf of S-GSC increases monotonously with the preset threshold $\gamma_{t h}$. The output mgf (12) can be written as

$$
\begin{aligned}
M_{s g}(s) & =L \sum_{n=0}^{L-1}\left(\begin{array}{c}
L-1 \\
n
\end{array}\right)(-1)^{n} \frac{1-e^{-\frac{\gamma_{t h}}{\bar{\gamma}}(1+n+\bar{\gamma} s)}}{1+n+\bar{\gamma} s} \\
& +\left[1-e^{-\frac{\gamma_{t h}}{\bar{\gamma}}}+\frac{e^{-\frac{\gamma_{t h}}{\bar{\gamma}}(1+s \bar{\gamma})}}{1+s \bar{\gamma}}\right]^{L}-\left[1-e^{-\frac{\gamma_{t h}}{\bar{\gamma}}}\right]^{L}
\end{aligned}
$$

Differentiating (28), we obtain

$$
\begin{aligned}
\frac{\partial M_{s g}(s)}{\partial \gamma_{t h}} & =\frac{L}{\bar{\gamma}} e^{-\frac{\gamma_{t h}}{\bar{\gamma}}(1+s \bar{\gamma})}\left(1-e^{-\frac{\gamma_{t h}}{\bar{\gamma}}}\right)^{L-1}+\frac{L}{\bar{\gamma}}\left[1-e^{-\frac{\gamma_{t h}}{\bar{\gamma}}}\right. \\
& \left.+\frac{e^{-\frac{\gamma_{t h}}{\bar{\gamma}}(1+s \bar{\gamma})}}{1+s \bar{\gamma}}\right]^{L-1} e^{-\frac{\gamma_{t h}}{\bar{\gamma}}}\left(1-e^{-s \gamma_{t h}}\right)-\frac{L}{\bar{\gamma}} e^{-\frac{\gamma_{t h}}{\bar{\gamma}}} \\
& \times\left(1-e^{-\frac{\gamma_{t h}}{\bar{\gamma}}}\right)^{L-1}\left(e^{\frac{\gamma_{t h}}{\bar{\gamma}}}-e^{-s \gamma_{t h}}\right) .
\end{aligned}
$$

For any given $\bar{\gamma}$ and real value $s>0, \frac{\partial M_{s g}(s)}{\partial \gamma_{t h}}>0$ and $M_{s g}(s)>0$, thus, $M_{s g}(s)$ increases monotonically with $\gamma_{t h}$. So does the SER, which is given by (4).

\section{REFERENCES}

[1] Y Roy, J-Y Chouinard and S. A. Mahmoud, "Selection diversity combining with multiple antennas for $m m$-wave indoor wireless channels," IEEE J. Select. Areas. Commun., vol. 14, no. 4, May 1996.

[2] L. Yue, "Analysis of generalized selection combining techniques," Proc. IEEE VTC 2000, vol. 2, pp. 1191-1195, 2000.

[3] M. -S. Alouini, M. K. Simon, "An MGF-based performance analysis of generalized selection combining over Rayleigh fading channels," IEEE Trans. Commun., vol. 48, pp. 401-415, Mar. 2000.

[4] A. Annanialai and C. Tellambura, "A new approach to performance evaluation of generalized selection diversity receivers in wireless channels," in IEEE Vehicular Technology Conference. IEEE, 2001, pp. 2309-2313.

[5] N. Kong, T. Eng, and L. B. Milstein, "A selection combining scheme for RAKE receivers," in IEEE ICUPC. IEEE, 1995, pp. 426-430.

[6] A. I. Sulyman and M. Kousa, "Bit error rate performance of a generalized diversity selection combining scheme in Nakagami fading channels," Proc. IEEE WCNC'00, vol. 3, pp. 1080-1085, 2000.
[7] M. K. Simon and M.-S. Alouini, "Performance analysis of generalized selection combining with threshold test per branch (T-GSC)," IEEE Trans. Veh. Technol., vol. 51, no. 5, pp. 1018-1029, Sept. 2002.

[8] C. Tellambura, A. J. Mueller and V. K. Bhargava, "Analysis of Mary phase-shift keying with diversity reception for land-mobile satellite channels," IEEE Trans. Veh. Technol., vol. 46, pp. 910-922, Nov. 1997.

[9] M. K. Simon and M.-S. Alouini, "A unified approach to the performance analysis of digital communication over generalized fading channels," Proc. IEEE, vol. 86, no. 9, pp. 1860-1877, Sept. 1998.

[10] G. L. Stuber, Principles of mobile communications. Norwell, MA: Kluwer, 1996

[11] J. G. Proakis, Digital Communications, 3rd ed. New York: McGraw-Hill, 1995.

[12] Y. Chen and C. Tellambura, "A new hybrid generalized selection combining scheme and its performance over fading channels," Submitted to IEEE Trans. Veh. Technol.

[13] U. Charash, "Reception through Nakagami multipath channels with random delays," IEEE Trans. Commun., vol. 27, no. 4, pp. 657-670, Apr. 1979.

[14] Mohamed-Slim Alouini, Marvin K. Simon, "Dual diversity over correlated log-normal fading channels," IEEE Trans. Commun., vol. 50, no. 12, pp. $1946-1959$, Dec. 2002.

[15] M. Win and J. Winters, "Analysis of hybrid selection/maximal-ratio combining in Rayleigh fading," IEEE Trans. Commun., vol. 47, pp. 17731776, Dec. 1999.

[16] W. C. Jakes, Microwave Mobile Communications. New York: IEEE Press, 1994. 